\title{
Bromination of graphene and graphite
}

\author{
A. Yaya, ${ }^{1}$ C. P. Ewels, ${ }^{1,}$ I. Suarez-Martinez, ${ }^{1,2}$ Ph. Wagner, ${ }^{1}$ S. Lefrant, ${ }^{1}$ A. Okotrub, ${ }^{3}$ L. Bulusheva, ${ }^{3}$ and P. R. Briddon ${ }^{4}$ \\ ${ }^{1}$ Institut des Matériaux Jean Rouxel, Université de Nantes, CNRS UMR 6502, FR-44322 Nantes, France \\ ${ }^{2}$ Nanochemistry Research Institute, Curtin University of Technology, Perth, WA 6845, Australia \\ ${ }^{3}$ Nikolaev Institute of Inorganic Chemistry SB RAS, 3 Acad. Lavrentiev Avenue, Russia \\ ${ }^{4}$ School of Natural Sciences, University of Newcastle upon Tyne, Newcastle upon Tyne, United Kingdom
}

(Received 14 December 2010; published 21 January 2011)

\begin{abstract}
We present a density-functional theory study of low-density bromination of graphene and graphite, finding significantly different behavior in these two materials. In graphene, we find a new $\mathrm{Br}_{2}$ form where the molecule sits perpendicular to the graphene sheet with an extremely strong molecular dipole. The resultant $\mathrm{Br}^{+}-\mathrm{Br}^{-}$has an empty $p_{z}$ orbital located in the graphene electronic $\pi$ cloud. Bromination opens a small (86-meV) band gap and strongly dopes the graphene. In contrast, in graphite, we find $\mathrm{Br}_{2}$ is most stable parallel to the carbon layers with a slightly weaker associated charge transfer and no molecular dipole. We identify a minimum stable $\mathrm{Br}_{2}$ concentration in graphite, finding low-density bromination to be endothermic. Graphene may be a useful substrate for stabilizing normally unstable transient molecular states.
\end{abstract}

DOI: 10.1103/PhysRevB.83.045411

PACS number(s): 73.22.Pr, 31.15.A-, 37.30.+i, 81.05.uf

\section{INTRODUCTION}

The intercalation of carbon nanomaterials with electron donors and acceptors is an active research area in which much effort is channeled toward the understanding and controlling of the electronic properties of graphene and graphite. Numerous potential applications such as sensors, electronic display panels, hydrogen storage, and supercapacitors ${ }^{1}$ have been suggested for such intercalated materials. The layered structure of graphites plays an important role in charge transfer reactions. Acceptor species can intercalate between graphitic layers, expanding the graphite with the resultant hybrids known as graphite intercalated compounds. ${ }^{2}$

Bromine acts as an acceptor when intercalated in materials such as graphite or nanotubes, and has been proposed experimentally as a way to open a band gap in three- or four-layer graphene. ${ }^{3}$ The in-plane electrical conductivity of graphite increases from $2.4 \times 10^{4} \Omega^{-1} \mathrm{~cm}^{-1}$ at room temperature to $2.2 \times 10^{5} \Omega^{-1} \mathrm{~cm}^{-1}$ after intercalation with bromine. $^{2}$

Bromine forms many ordered phases in graphites and undergoes an order-disorder phase transition as the amount of bromine or temperature changes. ${ }^{2,4-8}$ Bromine intercalated graphite forms stage- $n$ compounds, ${ }^{9}$ where $n$ is the number of graphitic layers between planes of $\mathrm{Br}_{2}(n>1)$. Extended $\mathrm{x}$-ray absorption fine structure (EXAFS) spectroscopy at room temperature and $100 \mathrm{~K}$ (Ref. 10) showed that intercalated bromine molecules lie parallel to the basal plane, with an expansion of the $\mathrm{Br}-\mathrm{Br}$ distance by $0.03 \AA$ to accommodate the lattice mismatch between the free molecule and the 2.46- $\AA$ spacing between graphite hexagons. The x-ray diffraction (XRD) and electron microscopy studies ${ }^{6}$ suggest that intercalated $\mathrm{Br}_{2}$ at lower concentration is composed of chains of $\mathrm{Br}_{2}$ molecules in which the intermolecular distance is identical to that of solid bromine.

Graphite Raman spectra associated with $\mathrm{Br}_{2}$ intercalation show a strong peak at $242 \mathrm{~cm}^{-1}$ (Refs. 11-14) assigned to the intercalated $\mathrm{Br}_{2}$ stretch mode. The frequency is downshifted from $320 \mathrm{~cm}^{-1}$ for gaseous $\mathrm{Br}_{2}$ and from $295 \mathrm{~cm}^{-1}$ for solid molecular bromine. ${ }^{15}$ There have been limited density-functional studies of brominated graphite ${ }^{16}$ and graphene. ${ }^{1,17}$

\section{METHOD}

In this paper, we examine low-density bromination of graphene and graphite using density-functional (DFT) calculations within the local density approximation (LDA). ${ }^{18}$ This method has been used successfully to study intercalated boron in graphite. ${ }^{19}$ A localized Gaussian basis set is used with a large number of fitting functions per atom ( 22 for each $\mathrm{C}$ atom and 50 for each $\mathrm{Br}$ atom), with angular momenta up to $l=2$ for $\mathrm{C}$ and $l=3$ for $\mathrm{Br}$. A finite-temperature electron level filling of $k T=0.04 \mathrm{eV}$ is used to improve convergence. Core electrons were eliminated using norm-conserving relativistic pseudopotentials of Hartwigsen, Goedecker, and Hutter. ${ }^{20} \mathrm{~A}$ cut-off energy of 150 Hartrees was used to obtain convergence of the charge density.

Isolated $\mathrm{Br}_{2}$ was calculated in a $13.23-\AA$ cubic supercell. Hexagonal $4 \times 4$ graphene supercells containing $\mathrm{C}_{32} \mathrm{Br}_{2}$ were used with a large vacuum spacing of $31 \AA$ between layers to ensure no interlayer interaction, and a $4 \times 4 \times 1$ MonkhorstPack $k$-point grid. ${ }^{21}$ Graphite calculations used $3 \times 3 \times n$ layer supercells $\left(\mathrm{C}_{18}\right)_{n}\left(\mathrm{Br}_{2}\right)_{m}(n=1-4$ and $m=1,2)$ for different layer stackings, with $4 \times 4 \times 1$ or $4 \times 4 \times 2 k$-point grids depending on cell size. All structures were fully geometrically optimized with no constraints of symmetry, allowing both atomic positions and cell dimensions to vary freely. Atomic charge states were obtained by summing Mulliken population analysis over all the filled electronic states. Vibrational frequencies were calculated by determining the energy and forces for \pm 0.2 -a.u. displacements of the Bromine atoms. The second derivatives on the displaced atoms can then be found from the two-sided difference formula for the second derivative. All results are spin averaged, and test calculations with spin polarization all gave zero spin solutions as the most thermodynamically stable.

Our calculated bond length, 2.29 quency, $326 \mathrm{~cm}^{-1}$, for isolated $\mathrm{Br}_{2}$ show excellent agreement 
with experiment $\left(2.27 \AA\right.$ and $323 \mathrm{~cm}^{-111}, 2.283 \AA$ and $320 \mathrm{~cm}^{-113}$ ) and literature DFT/LDA values $[2.263 \AA$ (Ref. 22), $2.244 \AA$, and $324 \mathrm{~cm}^{-11}$ ].

\section{BROMINE ON GRAPHENE}

Figure 1 shows the structures of $\mathrm{Br}_{2}$ over graphene in both perpendicular and parallel orientations after geometry optimization, with calculated properties for $\mathrm{Br}_{2}^{\perp}$ and $\mathrm{Br}_{2}^{\|}$ [structures (a) and (d), respectively, in Fig. 1] in Table I.

The most thermodynamically stable arrangement is $\mathrm{Br}_{2}$ oriented perpendicular to the graphene sheet above a carbon atom [Fig. 1(a), referred to hereafter as $\mathrm{Br}_{2}^{\perp}$ ]. Its binding energy of $0.40 \mathrm{eV}$ shows that it will be strongly physisorbed at room temperature. However, the small variations in binding energy between structures suggest that low-density $\mathrm{Br}_{2}$ binding to graphene will be largely orientation independent; at these densities at room temperature, $\mathrm{Br}_{2}$ should be in constant tumbling motion (this also holds for the results of Ref. 17). Increasing the $\mathrm{Br}_{2}$ concentration did not significantly change the relative energies of perpendicular and parallel orientations. However, it is possible to obtain twice the maximum surface density for $\mathrm{Br}_{2}^{\perp}$ as for $\mathrm{Br}_{2}$ in the parallel orientation [Fig. 1(d), referred to hereafter as $\mathrm{Br}_{2}^{\|}$). Thus, for the limit of high surface concentrations, we expect $\mathrm{Br}_{2}^{\perp}$ to dominate.

\section{A. $\mathrm{Br}_{2}$ perpendicular to graphene surface}

$\mathrm{Br}_{2}^{\perp}$ represents a very unusual configuration for bromine. It shows strong charge transfer $(0.129 e)$ from the graphene, with a very strong induced molecular dipole $\left(\mathrm{Br}^{+0.480}-\mathrm{Br}^{-0.609}\right)$. The singly occupied $p_{z}$ orbital of the lower $\mathrm{Br}$ atom depopulates into the $p_{z}$ of the upper $\mathrm{Br}$ atom, forming a nascent bromonium and bromide ion pair. In this way, the emptied lower $p_{z}$ orbital can sit within the graphene $\pi$ cloud $(\mathrm{Br}$ is only $2.74 \AA$ above the graphene).

This behavior is reminiscent of the well-known reaction between $\mathrm{Br}_{2}$ and unsaturated bonds in organic chemistry. However, in these cases, this dipolar form of $\mathrm{Br}_{2}$ is an unstable transient state that immediately saturates the $\mathrm{C}=\mathrm{C}$ bond, forming two $\mathrm{Br}-\mathrm{C}$ bonds. In graphene, this final step would be endothermic due to steric hindrance between the $\mathrm{Br}$ atoms as a result of the mechanical confinement of the lattice. Indeed, our attempts to stabilize $\mathrm{C}-\mathrm{Br}$ pairs on graphene in
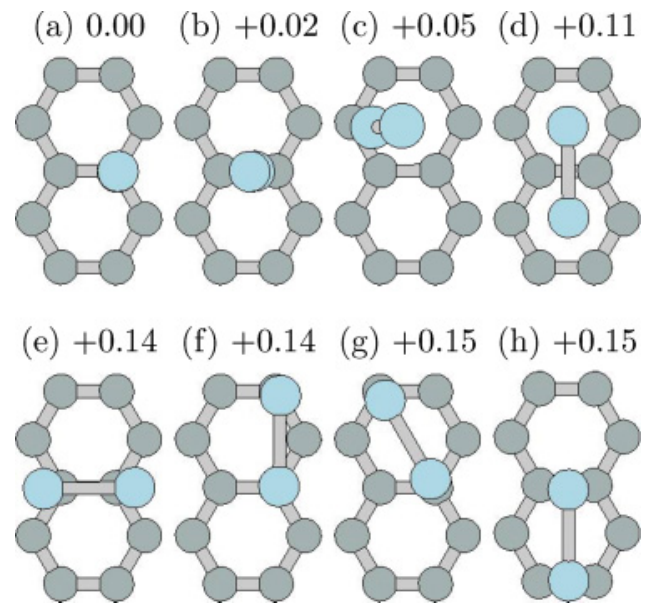

FIG. 1. (Color online) Optimized geometries of $\mathrm{Br}_{2}$ molecule (a)-(c) perpendicular and parallel (d)-(h) to a graphene sheet. Relative stabilities are quoted in $\mathrm{eV}$ [binding energy of $\mathrm{Br}_{2}$ in structure (a) is $0.40 \mathrm{eV}]$. The text refers to (a) $\mathrm{Br}_{2}^{\perp}$ and (d) $\mathrm{Br}_{2}^{\|}$.

both neighboring $(1,2)$ and cross-hexagon $(1,4)$ configurations both resulted in $\mathrm{Br}$ spontaneously reconstructing into a $\mathrm{Br}_{2}$ molecule [placing $\mathrm{Br}-\mathrm{C}$ in a $(1,4)$ configuration with $\mathrm{Br}$ atoms on opposite sides of the graphene is $1.68 \mathrm{eV}$ less stable than $\left.\mathrm{Br}_{2}^{\perp}\right]$.

The unusual $\mathrm{Br}_{2}^{\perp}$ configuration is reflected in the band structure [Fig. 2(a)]. The strong coupling between the graphene lowest unoccupied molecular orbital (LUMO) and the $\mathrm{Br}_{2}$ antibonded state at around $+0.6 \mathrm{eV}$ reflects the interaction between the empty $\mathrm{Br} p_{z}$ orbital and the graphene $\pi$ cloud, with the resultant low-density $\mathrm{Br}_{2}$ layer opening a small 86-meV band gap.

\section{B. $\mathrm{Br}_{2}$ parallel to graphene surface}

In contrast, $\mathrm{Br}_{2}^{\|}$has no induced dipole, with weaker charge transfer $(0.084 e)$ from the graphene. The molecule sits $3.254 \AA$ above the graphene, i.e., above the $\pi$ cloud, with the additional charge occupying the $\mathrm{Br}_{2} p p \sigma^{*}$ antibonding state. The band structure [Fig. 2(b)] shows that the $\mathrm{Br}_{2}$ states lie lower than those of $\mathrm{Br}_{2}^{\perp}$ by $\sim 0.7 \mathrm{eV}$. The $\mathrm{Br}_{2}$ antibonded state pins the Fermi level $\sim 0.2 \mathrm{eV}$ lower than in the pristine case, leaving graphene states around the $K$ point depopulated, indicating

TABLE I. Calculated parameters for the most stable $\mathrm{Br}_{2}^{\perp}$ and $\mathrm{Br}_{2}^{\|}$on graphene compared with literature DFT calculations.

\begin{tabular}{lccc}
\hline \hline & $\mathrm{Br}_{2}^{\perp}$ & $\mathrm{Br}_{2}^{\|}$ & Literature \\
\hline Orientation & Perp. & Parallel & Parallel \\
Binding energy $\left[\mathrm{Br}_{2}(\mathrm{eV})\right]$ & -0.40 & -0.29 & $-0.20^{1}$ \\
& & & $-0.29^{17}$ \\
$\mathrm{Br}-\mathrm{Br}$ stretch frequency $\left(\mathrm{cm}^{-1}\right)$ & 288 & 270 & $311^{1}$ \\
$\mathrm{Br}-\mathrm{Br}$ bond length $(\AA)$ & 2.33 & 2.31 & $2.245^{1}$ \\
$\mathrm{Br}-\mathrm{C}$ distance $(\AA)$ & 2.74 & 3.45 & $3.375^{1}$ \\
& & & $3.74^{17}$ \\
Charge state of $\mathrm{Br}$ atoms $(e)$ & +0.48 & -0.04 & \\
Total charge transfer $\left[\mathrm{Br}_{2}(e)\right]$ & -0.61 & -0.04 & \\
\hline \hline
\end{tabular}


(a)

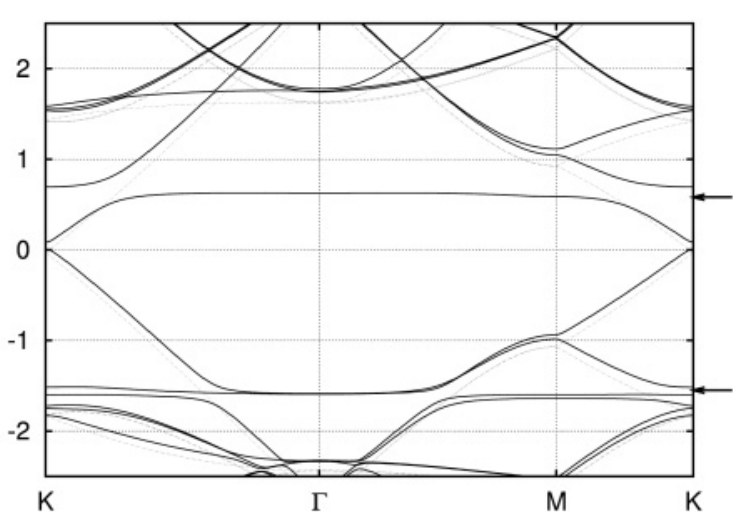

(b)

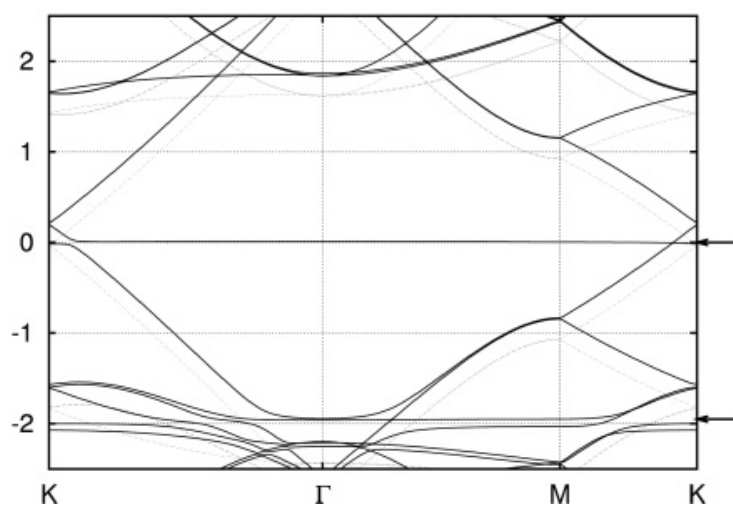

FIG. 2. Band structure of (a) $\mathrm{Br}_{2}^{\perp}$ and (b) $\mathrm{Br}_{2}^{\|}$on graphene (eV); faded dotted lines indicate the same supercell of pristine graphene for comparison. Arrows indicate bromine related states.

charge transfer from graphene to $\mathrm{Br}_{2}$. The bromine states are flat and largely decoupled from the graphene bands since there is only weak interaction between $\mathrm{Br}_{2}$ orbitals and the graphene $\pi$ cloud in this orientation.

\section{Spectroscopy of bromine on graphene}

Our results are consistent with experimental Raman observations of brominated graphene. ${ }^{3}$ For graphene with three or more layers, a resonant Raman signal was observed for $\mathrm{Br}_{2}$ at around $240 \mathrm{~cm}^{-1}$; for monolayer and bilayer graphene, no bromine signal was observed. This could be an orientation effect, since if the $\mathrm{Br}_{2}$ sits perpendicular to the surface as we propose and orthogonal Raman is used, then the molecules will be aligned with the beam and there will be no interaction and, hence, no signal. In addition, the bromine highest occupied molecular orbitals (HOMO) and LUMO states are further apart for $\mathrm{Br}_{2}^{\perp}(\sim 2.2 \mathrm{eV})$ than for $\mathrm{Br}_{2}^{\|}(\sim 1.96 \mathrm{eV})$ or for the various graphite structures we have examined $(1.7-2.0 \mathrm{eV})$. Given the excitation laser used $(633 \mathrm{~nm}, 1.96 \mathrm{eV}), \mathrm{Br}_{2}^{\perp}$ may not be in resonance as the authors suggested. ${ }^{3} \mathrm{Br}_{2}^{\|}$would have an associated Raman signal, and, since none is observed, this allows us to exclude this configuration. We note that the strong dipole of $\mathrm{Br}_{2}^{\perp}$ will make the molecule infrared active.

\section{BROMINE IN GRAPHITE}

We now turn our attention to graphite. Our calculated energy to separate $A B$ graphite layers is $36.7 \mathrm{meV} /$ atom, with $A A$-stacked graphite $12.0 \mathrm{meV} /$ atom less stable than $A B$-stacked graphite. These figures are in good agreement with experiment (35 meV/atom) (Ref. 23) and previous calculations (9.68-9.70 meV/atom $A A-A B$ energy difference). ${ }^{24,25}$ Our interlayer spacings of 3.39 and $3.50 \AA$ for $A B$ - and $A A$-stacked graphite, respectively, are also in reasonable agreement with previous calculations. ${ }^{25}$

We placed $\mathrm{Br}_{2}$ in a variety of different orientations and locations including above $\alpha$ - and $\beta$-carbon atom sites and hexagon centers in graphite of various layer stackings. Unlike graphene, $\mathrm{Br}_{2}$ in graphite is more stable parallel to the graphitic layers with $\mathrm{Br}$ atoms above hexagon centers (see Table II), in agreement with experiment. ${ }^{6,10}$ Perpendicular oriented $\mathrm{Br}_{2}$ structures are much less stable (by typically $0.52 \mathrm{eV}$ ). The results are summarized and compared with experiment in Table II. We note that the charge transfer value from Raman was reported with a large uncertainty. ${ }^{13}$

Aside from the lowest energy structures quoted here, we obtained many metastable structures. While their energies were all within $0.01-0.05 \mathrm{eV}$ of those structures discussed here, they show significant variation in the $\mathrm{Br}_{2}$ stretch frequency (250$278 \mathrm{~cm}^{-1}$ ), and slight variation in position and $\mathrm{Br}-\mathrm{Br}$ bond length (2.31-2.33 $\AA$ ). This suggests that, at room temperature, $\mathrm{Br}_{2}$ in graphite will be mobile and is consistent with the observation of a broad and somewhat complicated Raman peak. ${ }^{11}$

We explored all possible layer stacking combinations for stage-1 and stage- 2 intercalated graphites. Bromine molecules are most stable with $A A$-stacked graphite on each side, while unbrominated graphite layers preferentially are $A B$ stacked. Thus (indicating layers of bromine molecules by $X$, we find the most stable stage- 1 phase to be $[A X]_{n}$ and stage- 2 phase

TABLE II. Calculated and experimental results for $\mathrm{Br}_{2}$ intercalated graphite. The $\mathrm{C}-\mathrm{C}$ layer distance refers to the layers separated by bromine. Experimental values from EXAFS, ${ }^{10}$ Raman,,${ }^{11,13}$ and XRD. $., 9,12,26$ At these low concentrations, $\mathrm{Br}_{2}$ intercalation is endothermic.

\begin{tabular}{lccc}
\hline \hline & Stage 1 & Stage2 & Experiment \\
\hline Cell used & $\mathrm{C}_{18} \mathrm{Br}_{2}$ & $\mathrm{C}_{72} \mathrm{Br}_{4}$ & \\
Binding energy $\left[\mathrm{Br}_{2}(\mathrm{eV})\right]$ & +0.08 & +0.08 & \\
$\mathrm{C}-\mathrm{C}$ layer distance $(\AA)$ & 6.45 & 6.47 & $7.0^{9,12}, 7.05^{26}$ \\
$c$ axis $(\AA)$ & 9.80 & 9.82 & $10.7^{6}$ \\
$\mathrm{Br}-\mathrm{Br}$ bond length $(\AA)$ & 2.30 & 2.30 & $2.34^{10,12,27}$ \\
$\mathrm{Br}-\mathrm{C}$ distance $(\AA)$ & 3.51 & 3.46 & $2.9^{10}$ \\
$\mathrm{Br}-\mathrm{Br}$ frequency $\left(\mathrm{cm}^{-1}\right)$ & 287 & 274 & $242-258^{11,13}$ \\
Charge transfer $\left[\mathrm{Br}_{2}(e)\right]$ & -0.10 & -0.12 & $-0.16^{27}-0.34^{13}$ \\
\hline \hline
\end{tabular}


to be $[A X A B X B]_{n}$, suggesting $[A X A B]_{n}$ and $[A X A B A B X B A B]_{n}$ for stage 3 and stage 4 , respectively.

Our calculated bromine intercalation energy is weakly endothermic, since at these low densities, the energetic cost associated with separating graphite planes (a cost per unit area) is not sufficiently offset by the binding energy of $\mathrm{Br}_{2}$ to the layers. Subtracting the energy to separate graphite layers from the $\mathrm{Br}_{2}$ intercalation energy gives an energy for intercalation of $\mathrm{Br}_{2}$ into pre-separated stage- 1 graphite of $0.581 \mathrm{eV} / \mathrm{Br}_{2}$. This implies that the minimum $\mathrm{Br}_{2}$ concentration for exothermic intercalation in stage- $n$ graphite will be $\mathrm{C}_{16 n} \mathrm{Br}_{2}$. Indeed, a fixed- $\mathrm{Br}$ calculation for a $\mathrm{C}_{8} \mathrm{Br}_{2}$ stage-1 high-coverage structure gives $\mathrm{Br}_{2}$ intercalation as exothermic. Thus, $\mathrm{Br}_{2}$ will aggregate in the same interlayer space and should be considered as a layer rather than as individual molecules.

This minimum required concentration also suggests intercalation will be a slow diffusion process with an abrupt diffusion front. This is consistent with long experimental intercalation times,${ }^{28}$ despite the high bromine interlayer mobility. It also explains why, on out-gassing bromine, the material switches from a stage 2 to a compound to stage $n(n=3,4, \ldots)$ rather than remaining at stage 2 with a lower bromine density per layer. ${ }^{2,11,28}$

We note that frequency calculations incorporating the energetic double derivatives of surrounding carbon atoms gave identical values to within $1 \mathrm{~cm}^{-1}$, showing that the $\mathrm{Br}_{2}$ stretch mode is decoupled from the surrounding carbon lattice, consistent with the literature. ${ }^{1}$

Band-structure calculations of $\mathrm{Br}_{2}$ layers between graphite sheets (not shown here) give a bromine related state, which pins the Fermi level just below that of perfect graphite $(\sim 0.1 \mathrm{eV})$, indicating charge transfer from graphite to $\mathrm{Br}_{2}$. This state shows some dispersion indicating weak coupling with the underlying graphite. In other respects, the graphite band structures are barely perturbed.

\section{DISCUSSION AND CONCLUSIONS}

Our results on graphite and graphene can explain the anomalously large $G$ peak shift for single graphene sheets in comparison with few-layer graphene. ${ }^{3}$ The higher maximum $\mathrm{Br}_{2}$ surface density for $\mathrm{Br}_{2}^{\perp}$ than for interlayer $\mathrm{Br}_{2}$ means charge transfer per unit area will be higher. Additionally, $\mathrm{Br}_{2}$ can attach to both sides of graphene. We find a binding energy of $-0.38 \mathrm{eV} / \mathrm{Br}_{2}$ for two $\mathrm{Br}_{2}^{\perp}$ on either side of the same $\mathrm{C}$ atom, with an associated charge transfer of $-0.12 e / \mathrm{Br}_{2}$. Thus, the net total charge transfer per unit area will indeed be significantly higher for monolayer graphene than for multilayer systems.

Test calculations for a $(5,5)$ single-walled nanotube, either isolated or in bundles, gave similar structural behavior, i.e., $\mathrm{Br}_{2}$ on the surface of the isolated tube adopts a perpendicular orientation, while intercalated $\mathrm{Br}_{2}$ sits parallel to the tube walls. This will be explored further in a later publication.

In summary, we have examined low-density $\mathrm{Br}_{2}$ adsorption in graphene and graphite. In graphene, $\mathrm{Br}_{2}$ adopts an unusual perpendicular orientation, opening a small band gap ( $\sim 86 \mathrm{meV})$ in the graphene with strong charge transfer. The molecule forms a $\mathrm{Br}^{+}+\mathrm{Br}^{-}$pair, rendering it infrared active. This is a new form of $\mathrm{Br}_{2}$ previously only considered as an unstable intermediate to bromine-induced carbon-bond saturation. Such graphene-induced stabilization behavior may be mirrored in other molecular species, enabling the study of otherwise unstable reactive molecular forms.

In graphite, $\mathrm{Br}_{2}$ adopts a parallel orientation to the sheets with an associated charge transfer. Our calculations are in good agreement with experimental data, where available. At high bromine concentrations and low temperatures, there is some evidence of bromine chain structure formation ${ }^{29,30}$ in graphite, and we are currently investigating this further. We note that high-density bromination of graphite leads to stage-2 compounds, and, in conjunction with an appropriate secondary surfactant, this may be a promising way to produce bilayer graphene.

\section{ACKNOWLEDGMENT}

C.P.E. and P.W. thank the NANOSIM GRAPHENE (Project No. ANR-09-NANO-016-01) funded by the French National Agency (ANR) within the P3N2009 programme.

\footnotetext{
*Corresponding author: chris.ewels@ cnrs-imn.fr

${ }^{1}$ J. Seung-Hoon, S. G. Louie, and M. L. Cohen, Solid State Commun. 123, 495 (2002).

${ }^{2}$ M. S. Dresselhaus and G. Dresselhaus, Adv. Phys. 51, 1 (2002).

${ }^{3}$ N. Jung, N. Kim, S. Kockusch, N. J. Turro, P. Kim, and L. Brus, Nano Lett. 9, 4133 (2009).

${ }^{4}$ S. Bandow, G. Chen, G. U. Sumanasekera, R. Gupta, M. Yudasaka, S. Iijima, and P. C. Eklund, Phys. Rev. B 66, 075416 (2002).

${ }^{5}$ T. Yoichi, Y. Yasuko, A. Noboru, and S. Yashushi, J. Ceram. Soc. Jpn. 111, 42 (2003).

${ }^{6}$ W. T. Eeles and J. A. Turnbull, Proc. R. Soc. A 283, 179 (1965).

${ }^{7}$ B. Bach, M. Bagouin, F. Bloc, and A. Herold, C. R. Acad. Sci. (Paris) 257, 681 (1963).

${ }^{8}$ K. K. Bardhan, J. C. Wu, and D. D. L. Chung, Synth. Metals 2, 109 (1980).

${ }^{9}$ T. Sasa, Y. Takahashi, and T. Mukaibo, Carbon 9, 407 (1971).

${ }^{10}$ S. M. Heald and E. A. Stern, Phys. Rev. B 17, 4069 (1978).
}

${ }^{11}$ P. C. Eklund, N. Kambe, G. Dresselhaus, and M. S. Dresselhaus, Phys. Rev. B 18, 7069 (1978).

${ }^{12}$ A. Erbil, A. R. Kortan, R. J. Birgeneau, and M. S. Dresselhaus, Phys. Rev. B 28, 6329 (1983).

${ }^{13}$ A. Erbil, G. Dresselhaus, and M. S. Dresselhaus, Phys. Rev. B 25, 5451 (1982).

${ }^{14}$ D. Duan, Y. Liu, Y. Ma, Z. Liu, T. Cui, B. Liu, and G. Zou, Phys. Rev. B 76, 104113 (2007).

${ }^{15}$ J. E. Cahill and G. E. Leroi, J. Chem. Phys. 51, 4514 (1969).

${ }^{16}$ E. Widenkist et al., J. Phys. D: Appl. Phys. 42, 112003 (2009).

${ }^{17}$ A. N. Rudenko, F. J. Keil, M. I. Katsnelson, and A. I. Lichtenstein, Phys. Rev. B 82, 035427 (2010).

${ }^{18}$ P. R. Briddon and R. Jones, Phys. Status Solidi B 217, 131 (2000).

${ }^{19}$ I. Suarez-Martinez, A. A. El-Barbary, G. Savini, and M. I. Heggie, Phys. Rev. Lett. 98, 015501 (2007).

${ }^{20}$ C. Hartwigsen, S. Goedecker, and J. Hutter, Phys. Rev. B 58, 3641 (1998). 
${ }^{21}$ H. J. Monkhorst and J. D. Pack, Phys. Rev. B 13, 5188 (1976).

${ }^{22}$ M. S. Miao, V. E. Van Doren, and J. L. Martins, Phys. Rev. B 68, 094106 (2003).

${ }^{23}$ L. X. Benedict, N. G. Chopra, M. L. Cohen, A. Zettl, S. G. Louie, and V. H. Crespi, Chem. Phys. Lett. 286, 490 (1998).

${ }^{24}$ R. H. Telling and M. I. Heggie, Philos. Mag. Lett. 83, 411 (2003).
${ }^{25}$ J. C. Charlier, X. Gonze, and J. P. Michenaud, Europhys. Lett. 208, 403 (1994).

${ }^{26}$ W. Rudorff, Z. Anorg. Allg. Chem 245, 383 (1941).

${ }^{27}$ S. M. Heald and E. A. Stern, Synth. Metals 1, 249 (1980).

${ }^{28}$ J. E. Fischer, Acc. Chem. Res. 35, 1079 (2002).

${ }^{29}$ D. D. L. Chung, Phase transitions 8, 35 (1986).

${ }^{30}$ D. Chung, G. Dresselhaus, and M. S. Dresselhaus, Mater. Sci. Eng. 31, 107 (1977). 